\title{
Aggregating opinions in non-uniform ordered qualitative scales
}

\author{
José Luis García-Lapresta \\ PRESAD Research Group, BORDA Research Unit, IMUVA, Departamento de Economía \\ Aplicada, Universidad de Valladolid, Spain \\ David Pérez-Román \\ PRESAD Research Group, BORDA Research Unit, Departamento de Organización de \\ Empresas y Comercialización e Investigación de Mercados, Universidad de Valladolid, Spain
}

\begin{abstract}
This paper introduces a new voting system in the setting of ordered qualitative scales. The process is conducted in a purely ordinal way by considering an ordinal proximity measure that assigns an ordinal degree of proximity to each pair of linguistic terms of the qualitative scale. Once the agents assess the alternatives through the qualitative scale, the alternatives are ranked according to the medians of the ordinal degrees of proximity between the obtained individual assessments and the highest linguistic term of the scale. Since some alternatives may share the same median, an appropriate tie-breaking procedure is introduced. Some properties of the proposed voting system have been provided.
\end{abstract}

Keywords: group decision making; qualitative scales; ordinal proximity measures.

\section{Introduction}

Ordered qualitative scales are common in social sciences, engineering, computer sciences and other fields, because they are more appropriate than numerical scales for dealing with the vagueness and imprecision of human beings when evaluating different alternatives. Some ordered qualitative scales are uniform: the psychological proximity between each pair of consecutive terms of the scale

Email addresses: lapresta@eco.uva.es (José Luis García-Lapresta), david@emp.uva.es (David Pérez-Román)

Preprint submitted to Applied Soft Computing

May 21, 2017 
is the same, e.g. the scale \{'very bad', 'bad', 'regular', 'good', 'very good'\}. Usually, this is the case of Likert-type scales [20]. However, not all ordered qualitative scales are uniform. For instance, the scale \{'reject', 'major revision', 'minor revision', 'accept'\}, that some scientific journals use for evaluating papers, may be considered as non-uniform (see García-Lapresta and Pérez-Román [14] for empirical evidence).

Although ordered qualitative scales consist of vague linguistic terms, sometimes these terms are represented by exact numerical values. For instance, the International Association of Oenologists considers that each attribute of a wine is evaluated in an ordered qualitative scale of seven linguistic terms: \{'bad', 'mediocre', 'inadequate', 'passable', 'good', 'very good', 'excellent'\} and each term is associated with an integer number (see Balinski and Laraki [4]). In spite of the fact of this practice has been widely used in the literature (see, for instance, Franceschini and Romano [10] and Averkin et al. [1]), it is meaningless because different codifications of the same ordered qualitative scale could generate different outcomes when aggregating individual assessments (see Roberts [23] and Franceschini et al. [9], among others).

In order to capture the vagueness of ordered qualitative scales, some authors assign other cardinal objects, such as intervals of real numbers or fuzzy numbers, to the linguistic terms of the scale (see, for instance, Zadeh [24], Bass and Kwakernaak [6] and Chen and Hwang [7]). Again, these cardinal representations may be considered as meaningless.

Herrera and Martínez [17, 18] introduce the 2-tuple linguistic model for aggregating linguistic information in the setting of uniform ordered qualitative scales. The authors identify each linguistic term of the scale with its position in the scale; after an aggregation process, the outcome is represented by a pair (2-tuple) consisting of a linguistic term and a numerical value that measures the deviation with respect to the linguistic term. Thus, in practice, 2-tuples and real numbers are identical. The procedure is completed with a linear order on the set of 2-tuples that permits rank order the outcomes generated by the aggregation process. Although the procedure manages linguistic information, it is mathematically equivalent to work with numerical values (see García-Lapresta [11]).

Herrera et al. $[15,16]$ extend the 2-tuple linguistic model to the case of unbalanced qualitative scales by considering additional linguistic terms and under a high computational cost (see also Martínez and Herrera [22]). Bartczuk et. al. [5] modify the previous model by introducing numerical correction factors in 
the extended linguistic terms. This new model is computationally less expensive than the previous one and provides a simpler semantics.

In the mentioned approaches, the linguistic information and its aggregation are managed through cardinal objects and techniques. In this paper, we do not represent linguistic terms of ordered qualitative scales by any mathematical object. Instead, we consider psychological proximities among linguistic terms in a purely ordinal way, without using numerical distances, but ordinal degrees.

In real life, it is usual to make comparisons between proximities of different pairs of objects in a vague and ordinal fashion. For instance, we say "Rome is closer to Naples than to Milan", "Budapest is closer to Vienna than Paris is to Athens", etc. An excellent example of the use of ordinal proximities can be found in the following sentence of the Amos Oz' novel Suddenly in the Depth of the Forest: "... fairly close to Maya's back but not as close as she was to the stranger, and slightly closer than she was to the opening of the cave".

These kinds of ordinal comparisons will be taken into account in the setting of ordered qualitative scales through the notion of ordinal proximity measure, introduced by García-Lapresta and Pérez-Román [14] to deal with the psychological proximities among linguistic terms of ordered qualitative scales.

In the Majority Judgment (MJ) voting system, introduced by Balinski and Laraki $[2,3]$ ), agents evaluate the alternatives through the linguistic terms of an ordered qualitative scale. In MJ, the alternatives are ranked according to the medians of the obtained assessments. The authors also propose two different tie-breaking processes for obtaining the final ranking. Despite the fact that the qualitative scales considered by the authors are not necessarily uniform, the authors did not take this aspect into account.

In this paper, we use the new approach of ordinal proximity measures for designing a voting system that ranks the alternatives evaluated by the agents by means of an ordered qualitative scale. The proposed voting system is related to MJ, but we pay special attention to the ordinal proximities among the terms of the corresponding ordered qualitative scale. Concretely, alternatives are ranked according to the medians of the ordinal proximities between the individual assessments and the highest term of the scale. A tie-breaking procedure that takes into account the ordinal proximities among linguistic terms is also proposed. We also briefly show some properties of the devised voting system.

It should be noted that our approach shares with some soft computing methodologies the tolerance for imprecision, uncertainty and subjectivity, under a mathematical foundation (see Zadeh [25], Karray and De Silva [19] and 
Magdalena [21], among others).

The rest of the paper is organized as follows. Section 2 is devoted to ordinal proximity measures. In Section 3 we introduce and analyze the proposed voting system. Section 4 includes an example that illustrates how the voting system works. In Section 5 we extend the voting system to the case of multiple criteria. Finally, Section 6 concludes the paper with some remarks.

\section{Ordinal proximity measures}

We consider that each individual of a group of agents assigns a linguistic term to every feasible alternative. These linguistic terms belong to an ordered qualitative scale $\mathcal{L}=\left\{l_{1}, \ldots, l_{g}\right\}$, arranged from worst to best, $l_{1}<\cdots<l_{g}$, where the granularity of $\mathcal{L}$ is at least 3 , i.e., $g \geq 3$.

We now recall the notion of ordinal proximity measure, introduced by GarcíaLapresta and Pérez-Román [14]. It is a mapping that assigns an ordinal degree of proximity to each pair of linguistic terms of an ordered qualitative scale $\mathcal{L}$. These ordinal degrees of proximity belong to a linear order $\Delta=\left\{\delta_{1}, \ldots, \delta_{h}\right\}$, with $\delta_{1} \succ \cdots \succ \delta_{h}$, being $\delta_{1}$ and $\delta_{h}$ the maximum and minimum degrees of proximity, respectively. It is important noticing that the elements of $\Delta$ are not numbers. In fact, they are only abstract objects, without meaning, representing different degrees of proximity.

As usual in the setting of linear orders, $\delta_{r} \succeq \delta_{s}$ means $\delta_{r} \succ \delta_{s}$ or $\delta_{r}=\delta_{s}$; and $\delta_{r} \prec \delta_{s}$ means $\delta_{s} \succ \delta_{r}$.

Definition 1. ([14]) $A n$ ordinal proximity measure on $\mathcal{L}$ with values in $\Delta$ is a mapping $\pi: \mathcal{L}^{2} \longrightarrow \Delta$, where $\pi\left(l_{r}, l_{s}\right)=\pi_{r s}$ means the degree of proximity between $l_{r}$ and $l_{s}$, satisfying the following conditions:

1. Exhaustiveness: For every $\delta \in \Delta$, there exist $l_{r}, l_{s} \in \mathcal{L}$ such that $\delta=\pi_{r s}$.

2. Symmetry: $\pi_{s r}=\pi_{r s}$, for all $r, s \in\{1, \ldots, g\}$.

3. Maximum proximity: $\pi_{r s}=\delta_{1} \Leftrightarrow r=s$, for all $r, s \in\{1, \ldots, g\}$.

4. Monotonicity: $\pi_{r s} \succ \pi_{r t}$ and $\pi_{s t} \succ \pi_{r t}$, for all $r, s, t \in\{1, \ldots, g\}$ such that $r<s<t$.

We note that the previous conditions are independent (see García-Lapresta and Pérez-Román [14, Prop. 1]).

We say that an ordinal proximity measure $\pi: \mathcal{L}^{2} \longrightarrow \Delta$ is uniform if $\pi_{r(r+1)}=\pi_{s(s+1)}$ for all $r, s \in\{1, \ldots, g-1\}$, and totally uniform if $\pi_{r(r+t)}=$ $\pi_{s(s+t)}$ for all $r, s, t \in\{1, \ldots, g-1\}$ such that $r+t, s+t \leq g$. 
Each ordinal proximity measure $\pi: \mathcal{L}^{2} \longrightarrow \Delta$ will be represented by a $g \times g$ symmetric matrix with coefficients in $\Delta$, being the elements in the main diagonal $\pi_{r r}=\delta_{1}, r=1, \ldots, g$ :

$$
\left(\begin{array}{ccccc}
\pi_{11} & \cdots & \pi_{1 s} & \cdots & \pi_{1 g} \\
\cdots & \cdots & \cdots & \cdots & \cdots \\
\pi_{r 1} & \cdots & \pi_{r s} & \cdots & \pi_{r g} \\
\cdots & \cdots & \cdots & \cdots & \cdots \\
\pi_{g 1} & \cdots & \pi_{g s} & \cdots & \pi_{g g}
\end{array}\right) .
$$

This matrix will be called proximity matrix associated with $\pi$.

If we consider the conditions appearing in Definition 1, we would only need to show the upper half proximity matrix

$$
\left(\begin{array}{cccccc}
\delta_{1} & \pi_{12} & \pi_{13} & \cdots & \pi_{1(g-1)} & \pi_{1 g} \\
& \delta_{1} & \pi_{23} & \cdots & \pi_{2(g-1)} & \pi_{2 g} \\
& & & \cdots & \cdots & \cdots \\
& & & & \delta_{1} & \pi_{(g-1) g} \\
& & & & & \delta_{1}
\end{array}\right) .
$$

It is important noticing that the minimum proximity between linguistic terms is only reached when comparing the extreme linguistic terms: $\pi_{r s}=$ $\delta_{h} \Leftrightarrow(r, s) \in\{(1, g),(g, 1)\}$ (see García-Lapresta and Pérez-Román [14, Prop. 2]).

In the following example we illustrate an ordered qualitative scale of four linguistic terms with two extreme ordinal proximity measures.

Example 1. Consider $g=4$, where five ordinal degrees, not necessarily different, have to be assigned (see Fig. 1) and $h \in\{4,5,6,7\}$.

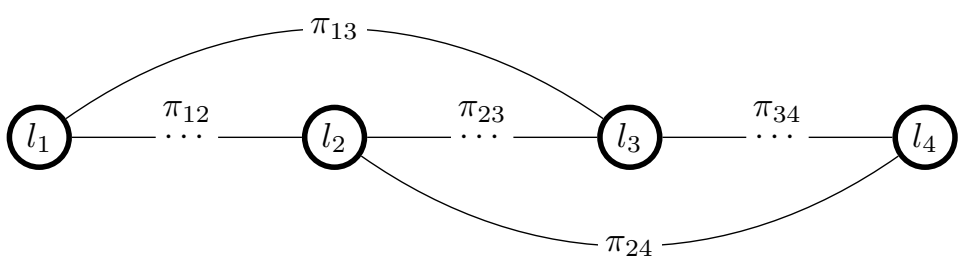

Figure 1: Ordinal degrees for $g=4$. 
It is worth mentioning that for $g=4$ there are 51 different ordinal proximity measures (García-Lapresta et al. [13]).

1. The simplest case corresponds to $\Delta=\left\{\delta_{1}, \delta_{2}, \delta_{3}, \delta_{4}\right\}$, with $\pi_{r r}=\delta_{1}$, $\pi_{12}=\pi_{23}=\pi_{34}=\delta_{2}, \pi_{13}=\pi_{24}=\delta_{3}$ and $\pi_{14}=\delta_{4}$, i.e., the totally uniform ordinal proximity measure, with associated matrix ${ }^{1}$

$$
A_{222}=\left(\begin{array}{cccc}
\delta_{1} & \delta_{2} & \delta_{3} & \delta_{4} \\
& \delta_{1} & \delta_{2} & \delta_{3} \\
& & \delta_{1} & \delta_{2} \\
& & & \delta_{1}
\end{array}\right)
$$

that can be visualized in Fig. 2 .

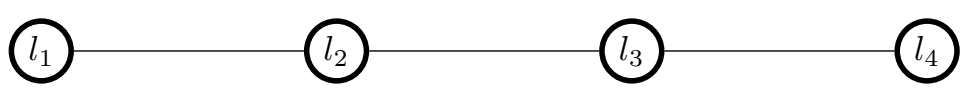

Figure 2: Ordinal proximity measure with associated matrix $A_{222}$.

2. We now consider $\Delta=\left\{\delta_{1}, \delta_{2}, \delta_{3}, \delta_{4}, \delta_{5}, \delta_{6}, \delta_{7}\right\}$, with $\pi_{r r}=\delta_{1}, \pi_{34}=\delta_{2}$, $\pi_{23}=\delta_{3}, \pi_{12}=\delta_{4}, \pi_{24}=\delta_{5}, \pi_{13}=\delta_{6}$ and $\pi_{14}=\delta_{7}$. In this case, the ordinal proximity measure has the following associated matrix

$$
A_{432}=\left(\begin{array}{cccc}
\delta_{1} & \delta_{4} & \delta_{6} & \delta_{7} \\
& \delta_{1} & \delta_{3} & \delta_{5} \\
& & \delta_{1} & \delta_{2} \\
& & & \delta_{1}
\end{array}\right)
$$

that can be visualized in Fig. 3 .

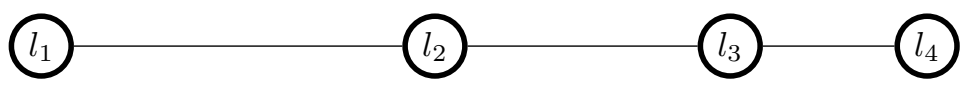

Figure 3: Ordinal proximity measure with associated matrix $A_{432}$.

\section{The voting system}

Consider a set of agents $A=\{1, \ldots, m\}$, with $m \geq 2$, that have to evaluate a set of alternatives $X=\left\{x_{1}, \ldots, x_{n}\right\}$, with $n \geq 2$, through an ordered qualitative

\footnotetext{
${ }^{1}$ The subindices 222 of the matrix $A_{222}$ correspond to the subindices of the $\delta$ 's appearing in the coefficients just over the main diagonal. We follow the same pattern in subsequent matrices.
} 
scale $\mathcal{L}=\left\{l_{1}, \ldots, l_{g}\right\}, l_{1}<\cdots<l_{g}$, with $g \geq 3$, and an ordinal proximity measure $\pi: \mathcal{L}^{2} \longrightarrow \Delta$.

The assessments provided by the agents to the alternatives are collected in a profile, that is a matrix

$$
V=\left(\begin{array}{ccccc}
v_{1}^{1} & \cdots & v_{i}^{1} & \cdots & v_{n}^{1} \\
\cdots & \cdots & \cdots & \cdots & \cdots \\
v_{1}^{a} & \cdots & v_{i}^{a} & \cdots & v_{n}^{a} \\
\cdots & \cdots & \cdots & \cdots & \cdots \\
v_{1}^{m} & \cdots & v_{i}^{m} & \cdots & v_{n}^{m}
\end{array}\right)
$$

that consists of $m$ rows and $n$ columns of linguistic terms, where the element $v_{i}^{a} \in \mathcal{L}$ is the linguistic assessment given by the agent $a \in A$ to the alternative $x_{i} \in X$.

\subsection{Ranking the alternatives}

To rank the alternatives, the procedure is divided in the following steps.

1. For each alternative $x_{i} \in X$, consider the assessments obtained by $x_{i}$ for all the agents: $v_{i}^{1}, \ldots, v_{i}^{m} \in \mathcal{L}($ column $i$ of $V)$.

2. For each alternative $x_{i} \in X$, calculate the ordinal proximities between the assessments obtained by $x_{i}$ and the highest linguistic term $l_{g}$ :

$$
\pi\left(v_{i}^{1}, l_{g}\right), \ldots, \pi\left(v_{i}^{m}, l_{g}\right) \in \Delta .
$$

In a different setting, Falcó et al. [8] rank order linguistic assessments taking into account their distances to the highest linguistic term of the ordered qualitative scale (the less, the better). However, in the present approach, when considering ordinal proximities between linguistic assessments and the highest linguistic term of the ordered qualitative scale, the pattern is just the opposite (the more, the better), because the notions of distance and proximity are antonyms.

3. For each alternative $x_{i} \in X$, arrange the previous ordinal degrees in a decreasing fashion and select the median $(\mathrm{s})^{2}, M_{i}$ :

\footnotetext{
${ }^{2}$ When the number of elements is odd, the median is unique. However, if that number
} 
(a) If the number of assessments is odd, then we duplicate the median. Thus, $M_{i}=\left(\delta_{r}, \delta_{r}\right)$ for some $r \in\{1, \ldots, h\}$.

(b) If the number of assessments is even, then we take into account the two medians. Thus, $M_{i}=\left(\delta_{r}, \delta_{s}\right)$ for some $r, s \in\{1, \ldots, h\}$ such that $r \leq s$.

Consequently, $M_{i} \in \Delta_{2}$, where $\Delta_{2}$ is the set of feasible medians:

$$
\Delta_{2}=\left\{\left(\delta_{r}, \delta_{s}\right) \in \Delta^{2} \mid r \leq s\right\}
$$

In the MJ voting system, Balinski and Laraki $[2,3]$ consider the lower median of the linguistic individual assessments as collective grade of each alternative when the number of assessments is odd (in MJ the individual assessments are arranged in an increasing manner). Choosing the lower median is not problematic when the number of agents is high, as happens in political elections. However, it can be considered as arbitrary when that number is low, as happens in small size committees.

In order to avoid loss of information, it is convenient to take into account the two medians. This requires to rank order feasible medians in a suitable way. For example, in our setting, $\left(\delta_{2}, \delta_{3}\right)$ is clearly better than $\left(\delta_{3}, \delta_{3}\right)$, and $\left(\delta_{3}, \delta_{3}\right)$ can be considered better than $\left(\delta_{2}, \delta_{4}\right)$ because $3+3=2+4$, but the dispersion (measured through the range of the subindices) is smaller in the first case than in the second one $(3-3=0<2=4-2)$.

In the next step we propose an appropriate linear order on the set of feasible medians.

4. To order the medians of ordinal proximities obtained by different alternatives in the previous step, consider the linear order $\succeq$ on $\Delta_{2}$ defined as

$$
\left(\delta_{r}, \delta_{s}\right) \succeq\left(\delta_{t}, \delta_{u}\right) \Leftrightarrow\left\{\begin{array}{l}
r+s<t+u \\
\text { or } \\
r+s=t+u \text { and } s-r \leq u-t,
\end{array}\right.
$$

for all $\left(\delta_{r}, \delta_{s}\right),\left(\delta_{t}, \delta_{u}\right) \in \Delta_{2}$.

is even, then there exist two medians. When the elements of a list are real numbers, the median of that list is usually defined as the arithmetic mean of the two medians. That it is impossible when the elements of the list are abstract objects, as happens when considering ordinal proximities. 
It is easy to see that if $r+s=t+u$, then $s-r \leq u-t \Leftrightarrow r \geq t \Leftrightarrow s \leq u$. Notice that $\left(\delta_{r}, \delta_{r}\right) \succeq\left(\delta_{t}, \delta_{t}\right) \Leftrightarrow r \leq t$.

5. Finally, the alternatives are ranked according to the weak order $\succcurlyeq$ on $X$ defined as $x_{i} \succcurlyeq x_{j} \Leftrightarrow M_{i} \succeq M_{j}$.

\subsection{Breaking ties}

Since some alternatives can share the same median(s), it is necessary to devise a tie-breaking process for ordering the alternatives. We propose to use a sequential procedure based on Balinski and Laraki [2] (see Balinski and Laraki [4] for practical examples). It consists of dropping the median(s) of the respective alternatives that are in a tie, and then select the new median(s) of the remaining ordinal degrees for the corresponding alternatives and applying the procedure given in (1).

Formally, when $M_{i}=M_{j}$ :

- If $m$ is odd, let $M_{i}^{(1)}, M_{j}^{(1)} \in \Delta_{2}$ be the medians obtained after dropping in $\pi\left(v_{i}^{1}, l_{g}\right), \ldots, \pi\left(v_{i}^{m}, l_{g}\right)$ and $\pi\left(v_{j}^{1}, l_{g}\right), \ldots, \pi\left(v_{j}^{m}, l_{g}\right)$ the ordinal degree appearing in $M_{i}=M_{j}$, respectively.

- If $m$ even, let $M_{i}^{(1)}, M_{j}^{(1)} \in \Delta_{2}$ be the medians obtained after dropping in $\pi\left(v_{i}^{1}, l_{g}\right), \ldots, \pi\left(v_{i}^{m}, l_{g}\right)$ and $\pi\left(v_{j}^{1}, l_{g}\right), \ldots, \pi\left(v_{j}^{m}, l_{g}\right)$ the pair of ordinal degrees appearing in $M_{i}=M_{j}$, respectively.

Then, the procedure given in (1) is applied again. If $M_{i}^{(1)}=M_{j}^{(1)}$, then the process continues with the remaining ordinal degrees for the corresponding alternatives until the ties are broken ${ }^{3}$. It is important noticing that alternatives with different assessments never become in a final tie.

\subsection{Properties}

We now enumerate some properties that the proposed voting system satisfies.

1. Anonymity: All individuals are treated in the same way.

2. Neutrality: All alternatives are treated in the same way.

3. Independence of irrelevant alternatives: The ranking between two alternatives only depends on the individual assessments obtained by these alternatives, being irrelevant the assessments obtained by other alternatives.

\footnotetext{
${ }^{3}$ Notice that in the following steps, the number of ordinal degrees is always even.
} 
4. Unanimity: If all agents assign the same or a better assessment to an alternative than to another one, then the second alternative cannot be ranked ahead the first one.

5. Monotonicity: Given two profiles with the only difference that an alternative receives a better assessment from an agent in the second profile, then that alternative cannot be ranked worse in the the second profile than in the first one.

6. Replication invariance: If all agents are replicated a number of times with the same assessments, then the outcome does not change.

\section{An illustrative example}

Consider five agents assessing the alternatives of $X=\left\{x_{1}, x_{2}, x_{3}\right\}$ through the ordered qualitative scale $\mathcal{L}=\left\{l_{1}, l_{2}, l_{3}, l_{4}\right\}$ and the profile

$$
\left(\begin{array}{ccc}
v_{1}^{1} & v_{2}^{1} & v_{3}^{1} \\
v_{1}^{2} & v_{2}^{2} & v_{3}^{2} \\
v_{1}^{3} & v_{2}^{3} & v_{3}^{3} \\
v_{1}^{4} & v_{2}^{4} & v_{3}^{4} \\
v_{1}^{5} & v_{2}^{5} & v_{3}^{5}
\end{array}\right)=\left(\begin{array}{lll}
l_{3} & l_{2} & l_{1} \\
l_{1} & l_{3} & l_{4} \\
l_{3} & l_{3} & l_{2} \\
l_{4} & l_{3} & l_{3} \\
l_{3} & l_{3} & l_{4}
\end{array}\right) .
$$

In Table 1 the number of linguistic terms obtained for each alternative is summarized.

\begin{tabular}{c|ccc} 
& $x_{1}$ & $x_{2}$ & $x_{3}$ \\
\hline$l_{1}$ & 1 & & 1 \\
$l_{2}$ & & 1 & 1 \\
$l_{3}$ & 3 & 4 & 1 \\
$l_{4}$ & 1 & & 2
\end{tabular}

Table 1: Number of linguistic terms obtained for each alternative.

In order to show that the ordinal proximity measure matters, we provide three different cases.

1. Consider the ordinal proximity measure with associated matrix $A_{222}$ appearing in Example 1. 
The ordinal proximities between the assessments obtained by the alternatives and $l_{4}$ are included in Table 2 .

\begin{tabular}{c|ccccc} 
& $\pi\left(v_{i}^{1}, l_{4}\right)$ & $\pi\left(v_{i}^{2}, l_{4}\right)$ & $\pi\left(v_{i}^{3}, l_{4}\right)$ & $\pi\left(v_{i}^{4}, l_{4}\right)$ & $\pi\left(v_{i}^{5}, l_{4}\right)$ \\
\hline$x_{1}$ & $\delta_{2}$ & $\delta_{4}$ & $\delta_{2}$ & $\delta_{1}$ & $\delta_{2}$ \\
$x_{2}$ & $\delta_{3}$ & $\delta_{2}$ & $\delta_{2}$ & $\delta_{2}$ & $\delta_{2}$ \\
$x_{3}$ & $\delta_{4}$ & $\delta_{1}$ & $\delta_{3}$ & $\delta_{2}$ & $\delta_{1}$
\end{tabular}

Table 2: Ordinal proximities between assessments and $l_{4}$.

We now arrange the previous ordinal degrees in a decreasing fashion:

$$
x_{1}: \delta_{1}, \delta_{2}, \delta_{2}, \delta_{2}, \delta_{4} ; \quad x_{2}: \delta_{2}, \delta_{2}, \delta_{2}, \delta_{2}, \delta_{3} ; \quad x_{3}: \delta_{1}, \delta_{1}, \delta_{2}, \delta_{3}, \delta_{4} .
$$

The three alternatives have the same median, $\delta_{2}$, i.e., $M_{1}=M_{2}=M_{3}=$ $\left(\delta_{2}, \delta_{2}\right)$. Then, it is necessary to use the tie-breaking process. After removing the median, we obtain

$$
x_{1}: \delta_{1}, \delta_{2}, \delta_{2}, \delta_{4} ; \quad x_{2}: \delta_{2}, \delta_{2}, \delta_{2}, \delta_{3} ; \quad x_{3}: \delta_{1}, \delta_{1}, \delta_{3}, \delta_{4} .
$$

The new medians are $M_{1}^{(1)}=M_{2}^{(1)}=\left(\delta_{2}, \delta_{2}\right)$ and $M_{3}^{(1)}=\left(\delta_{1}, \delta_{3}\right)$. By (1), $M_{1}^{(1)}=M_{2}^{(1)} \succ M_{3}^{(1)}$; then, $x_{1} \succ x_{3}$ and $x_{2} \succ x_{3}$. We use again the tie-breaking process with $x_{1}$ and $x_{2}$; thus, we remove the medians and obtain

$$
x_{1}: \delta_{1}, \delta_{4} ; \quad x_{2}: \delta_{2}, \delta_{3} .
$$

The new medians are $M_{1}^{(2)}=\left(\delta_{1}, \delta_{4}\right)$ and $M_{2}^{(2)}=\left(\delta_{2}, \delta_{3}\right)$. By (1), $M_{2}^{(2)} \succ M_{1}^{(2)}$; then, $x_{2} \succ x_{1}$ and, finally, we have $x_{2} \succ x_{1} \succ x_{3}$.

2. We now consider the ordinal proximity measure with associated matrix

$$
A_{223}=\left(\begin{array}{cccc}
\delta_{1} & \delta_{2} & \delta_{4} & \delta_{6} \\
& \delta_{1} & \delta_{2} & \delta_{5} \\
& & \delta_{1} & \delta_{3} \\
& & & \delta_{1}
\end{array}\right)
$$

that can be visualized in Fig. 4 . 


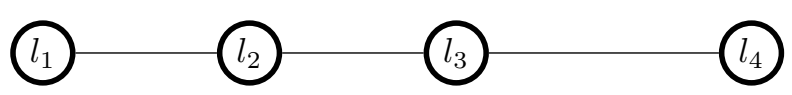

Figure 4: Ordinal proximity measure with associated matrix $A_{223}$.

The ordinal proximities between the assessments obtained by the alternatives and $l_{4}$ are included in Table 3 .

\begin{tabular}{c|ccccc} 
& $\pi\left(v_{i}^{1}, l_{4}\right)$ & $\pi\left(v_{i}^{2}, l_{4}\right)$ & $\pi\left(v_{i}^{3}, l_{4}\right)$ & $\pi\left(v_{i}^{4}, l_{4}\right)$ & $\pi\left(v_{i}^{5}, l_{4}\right)$ \\
\hline$x_{1}$ & $\delta_{3}$ & $\delta_{6}$ & $\delta_{3}$ & $\delta_{1}$ & $\delta_{3}$ \\
$x_{2}$ & $\delta_{5}$ & $\delta_{3}$ & $\delta_{3}$ & $\delta_{3}$ & $\delta_{3}$ \\
$x_{3}$ & $\delta_{6}$ & $\delta_{1}$ & $\delta_{5}$ & $\delta_{3}$ & $\delta_{1}$
\end{tabular}

Table 3: Ordinal proximities between assessments and $l_{4}$.

We now arrange the previous ordinal degrees in a decreasing fashion:

$$
x_{1}: \delta_{1}, \delta_{3}, \delta_{3}, \delta_{3}, \delta_{6} ; \quad x_{2}: \delta_{3}, \delta_{3}, \delta_{3}, \delta_{3}, \delta_{5} ; \quad x_{3}: \delta_{1}, \delta_{1}, \delta_{3}, \delta_{5}, \delta_{6} .
$$

The three alternatives have the same median, $\delta_{3}$, i.e., $M_{1}=M_{2}=M_{3}=$ $\left(\delta_{3}, \delta_{3}\right)$. After applying the tie-breaking process, the new medians are $M_{1}^{(1)}=M_{2}^{(1)}=\left(\delta_{3}, \delta_{3}\right)$ and $M_{3}^{(1)}=\left(\delta_{1}, \delta_{5}\right)$. By $(1), M_{1}^{(1)}=M_{2}^{(1)} \succ$ $M_{3}^{(1)}$; then, $x_{1} \succ x_{3}$ and $x_{2} \succ x_{3}$. Applying the tie-breaking process on $x_{1}$ and $x_{2}$, we obtain $M_{1}^{(2)}=\left(\delta_{1}, \delta_{6}\right)$ and $M_{2}^{(2)}=\left(\delta_{3}, \delta_{5}\right)$; then, by $(1)$, we have $x_{1} \succ x_{2} \succ x_{3}$.

3. We now consider the ordinal proximity measure with associated matrix

$$
A_{224}=\left(\begin{array}{cccc}
\delta_{1} & \delta_{2} & \delta_{3} & \delta_{6} \\
& \delta_{1} & \delta_{2} & \delta_{5} \\
& & \delta_{1} & \delta_{4} \\
& & & \delta_{1}
\end{array}\right)
$$

that can be visualized in Fig. 5 .

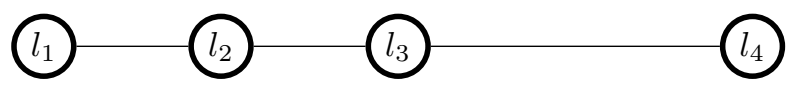

Figure 5: Ordinal proximity measure with associated matrix $A_{224}$. 
The ordinal proximities between the assessments obtained by the alternatives and $l_{4}$ are included in Table 4 .

\begin{tabular}{c|ccccc} 
& $\pi\left(v_{i}^{1}, l_{4}\right)$ & $\pi\left(v_{i}^{2}, l_{4}\right)$ & $\pi\left(v_{i}^{3}, l_{4}\right)$ & $\pi\left(v_{i}^{4}, l_{4}\right)$ & $\pi\left(v_{i}^{5}, l_{4}\right)$ \\
\hline$x_{1}$ & $\delta_{4}$ & $\delta_{6}$ & $\delta_{4}$ & $\delta_{1}$ & $\delta_{4}$ \\
$x_{2}$ & $\delta_{5}$ & $\delta_{4}$ & $\delta_{4}$ & $\delta_{4}$ & $\delta_{4}$ \\
$x_{3}$ & $\delta_{6}$ & $\delta_{1}$ & $\delta_{5}$ & $\delta_{4}$ & $\delta_{1}$
\end{tabular}

Table 4: Ordinal proximities between assessments and $l_{4}$.

We now arrange the previous ordinal degrees in a decreasing fashion:

$$
x_{1}: \delta_{1}, \delta_{4}, \delta_{4}, \delta_{4}, \delta_{6} ; \quad x_{2}: \delta_{4}, \delta_{4}, \delta_{4}, \delta_{4}, \delta_{5} ; \quad x_{3}: \delta_{1}, \delta_{1}, \delta_{4}, \delta_{5}, \delta_{6}
$$

The three alternatives have the same median, $\delta_{4}$, i.e., $M_{1}=M_{2}=M_{3}=$ $\left(\delta_{4}, \delta_{4}\right)$. After applying the tie-breaking process, the new medians are $M_{1}^{(1)}=M_{2}^{(1)}=\left(\delta_{4}, \delta_{4}\right)$ and $M_{3}^{(1)}=\left(\delta_{1}, \delta_{5}\right)$. By $(1)$, we have $M_{3}^{(1)} \succ$ $M_{1}^{(1)}=M_{2}^{(1)}$; then, $x_{3} \succ x_{1}$ and $x_{3} \succ x_{2}$. By using again the tiebreaking process with $x_{1}$ and $x_{2}$, the new medians are $M_{1}^{(2)}=\left(\delta_{1}, \delta_{6}\right)$ and $M_{2}^{(2)}=\left(\delta_{4}, \delta_{5}\right)$. Since $M_{1}^{(2)} \succ M_{2}^{(2)}$, we finally obtain $x_{3} \succ x_{1} \succ x_{2}$.

It is important emphasizing that the three ordinal proximity measures generate different rankings on the set of alternatives. One of the reasons is that the ordinal proximities between $l_{3}$ and $l_{4}$ are $\delta_{2}, \delta_{3}$ and $\delta_{4}$, respectively; and the ordinal proximities between $l_{2}$ and $l_{4}$ are $\delta_{3}, \delta_{5}$ and $\delta_{5}$, respectively. This means that the assessments have different "value" in each case.

If we apply MJ, in the proposal of Balinski and Laraki [3], to the considered profile, the outcome is $x_{1} \sim x_{2} \succ x_{3}$, different to the ones obtained in the three cases we have analyzed.

\section{Extensions}

García-Lapresta and González del Pozo [12] devise a multi-criteria decisionmaking procedure in the context of uniform qualitative scales. In their proposal, agents evaluate the alternatives regarding several criteria by assigning one or two consecutive terms of the scale to each alternative in each criterion. Weights 
assigned to criteria are managed through replications of the corresponding ratings, and alternatives are ranked according to the medians of their ratings after the replications.

It is easy to check that the voting system proposed in this paper coincides with the one given in García-Lapresta and González del Pozo [12] when agents assign a single linguistic term to each alternative, only a criterion is considered and the qualitative scale is equipped with the corresponding totally uniform ordinal proximity measure.

It is also easy to extend the voting system proposed in this paper to the cases where there are multiple criteria and agents are allowed to assign one or two consecutive terms of a qualitative scale equipped with a non necessarily uniform ordinal proximity measure. To this purpose, it would be necessary to duplicate each proximity $\pi\left(l_{r}, l_{g}\right)$ when a single linguistic term $l_{r}$ is assigned, and consider the two proximities $\pi\left(l_{r}, l_{g}\right)$ and $\pi\left(l_{r+1}, l_{g}\right)$ when two consecutive linguistic terms, $l_{r}$ and $l_{r+1}$ are assigned.

Since distinct criteria may have different importance in the global decision, we consider a weighting vector $\boldsymbol{w}=\left(w_{1}, \ldots, w_{q}\right) \in[0,1]^{q}$, with $w_{1}+\cdots+w_{q}=$ 1 , where $C=\left\{c_{1}, \ldots, c_{q}\right\}$ is the set of criteria. For practical reasons, we assume that these weights have at most two decimals, i.e., the percentages $100 \cdot w_{1}, \ldots, 100 \cdot w_{q}$ are integer numbers.

The criteria weighting scheme should follow the replication proposal given by García-Lapresta and González del Pozo [12]: the profiles associated with the criteria are replicated according to the corresponding percentages, $100 \cdot w_{1}$, $\ldots, 100 \cdot w_{q}$. In practice, it should be convenient to calculate the greatest common divisor (gcd) of percentages associated with the weights, and divide each percentage by the gcd. Then, the minimum number of replications of each profile is obtained.

Example 2. Consider that five agents assess the alternatives of $X=\left\{x_{1}, x_{2}, x_{3}\right\}$ regarding the criteria of $C=\left\{c_{1}, c_{2}, c_{3}\right\}$, with associated weighting vector $\boldsymbol{w}=(0.3,0.3,0.4)$, through the ordered qualitative scale $\mathcal{L}=\left\{l_{1}, l_{2}, l_{3}, l_{4}\right\}$ equipped with the ordinal proximity measure with associated matrix $A_{223}$.

Consider the profiles $V_{1}, V_{2}$ and $V_{3}$ corresponding to the criteria $c_{1}, c_{2}$ and 
$c_{3}$, respectively:

$$
V_{1}=\left(\begin{array}{ccc}
l_{3} & l_{2} & l_{1} \\
l_{1} & l_{3} & l_{4} \\
l_{3} & l_{3} & l_{2} \\
l_{4} & l_{3} & l_{3} \\
l_{3} & l_{3} & l_{4}
\end{array}\right), \quad V_{2}=\left(\begin{array}{ccc}
l_{4} & l_{2} & l_{1} \\
l_{3} & l_{4} & l_{4} \\
l_{4} & l_{1} & l_{3} \\
l_{4} & l_{3} & l_{1} \\
l_{1} & l_{4} & l_{1}
\end{array}\right), \quad V_{3}=\left(\begin{array}{ccc}
l_{1} & l_{2} & l_{1} \\
l_{1} & l_{2} & l_{4} \\
l_{3} & l_{1} & l_{3} \\
l_{4} & l_{1} & l_{1} \\
l_{1} & l_{2} & l_{4}
\end{array}\right) .
$$

Taking into account the percentages $100 \cdot w_{1}=30,100 \cdot w_{2}=30$ and $100 \cdot w_{3}=40$, since $\operatorname{gcd}(30,30,40)=10$, the profiles corresponding to each criterion should be replicated $30 / 10=3,30 / 10=3$ and $40 / 10=4$ times, respectively.

After some computations, in the first step we obtain the following medians: $M_{1}=M_{3}=\left(\delta_{3}, \delta_{3}\right)$ and $M_{2}=\left(\delta_{5}, \delta_{5}\right)$. By (1), $M_{1}=M_{3} \succ M_{2}$; then, $x_{1} \succ x_{2}$ and $x_{3} \succ x_{2}$. Then, it is necessary to use the tie-breaking process. After removing the medians, the new medians are $M_{1}^{(1)}=M_{3}^{(1)}=\left(\delta_{3}, \delta_{3}\right)$ and by using again the tie-breaking process we obtain $M_{1}^{(2)}=\left(\delta_{3}, \delta_{3}\right)$ and $M_{3}^{(2)}=\left(\delta_{3}, \delta_{5}\right)$. Since $M_{1}^{(2)} \succ M_{3}^{(2)}$, we finally have $x_{1} \succ x_{3} \succ x_{2}$.

\section{Concluding remarks}

This paper proposes a voting system in the setting of ordered qualitative scales non-necessarily uniform. The novelty of the proposal lies on the purely ordinal approach, where the ordinal proximities among the linguistic terms of the qualitative scale are essential for obtaining the ranking on the set of alternatives generated by the individual assessments. This ranking is based on the median(s) of the ordinal proximities between the individual assessments and the highest linguistic term of the scale, through an appropriate linear order on the set of feasible medians.

Although this aggregation procedure only takes into account the proximities among the individual assessments and the highest linguistic term of the scale, all the ordinal proximities among the linguistic terms of the qualitative scale are relevant. This is due to the fact that the degree of proximity between each linguistic term and the highest linguistic term of the scale depends on the rest of ordinal comparisons, as shown in the illustrative example included in Section 4.

In the aggregation procedure and in the proposed tie-breaking process, when the number of corresponding assessments is even, we have considered the two 
medians. This avoids loss of information and it is also a novelty with respect to other ordinal approaches $([2,3])$.

As shown in Section 4, given an ordered qualitative scale, the outcome of the voting system could depend on the ordinal proximity measure fixed for describing the proximities among the linguistic terms of the scale. Then, a relevant problem is how to determine what is the most appropriate ordinal proximity measure in that scale. It is not a trivial problem and the solution may depend on the society where the voting system is applied. If several experts provide their opinions about the mentioned proximities, then an aggregation procedure is needed. This issue has been analyzed in García-Lapresta et al. [13].

The properties included in Subsection 3.3 ensure that the proposed voting system is suitable for group decision making applications in the setting of ordered qualitative scales.

\section{Acknowledgments.}

The authors are grateful to Tomasa Calvo and three anonymous referees for their helpful comments and suggestions. The financial support of the Spanish Ministerio de Economía y Competitividad (project ECO2016-77900-P) and ERDF are acknowledged.

\section{References}

[1] A.N. Averkin, O.P. Kuznetsov, A.A. Kulinich, N.V. Titova, Decisionmaking support in weakly structured subject domains: Analysis of situations and evaluation of alternatives, Journal of Computer and Systems Sciences International 45 (2006) 469-479.

[2] M. Balinski, R. Laraki, A theory of measuring, electing and ranking, Proceedings of the National Academy of Sciences of the United States of America 104 (2007) 8720-8725.

[3] M. Balinski, R. Laraki, Majority Judgment. Measuring, Ranking, and Electing, The MIT Press, Cambridge, MA, 2011.

[4] M. Balinski, R. Laraki, How best to rank wines: Majority Judgment, in: Wine Economics: Quantitative Studies and Empirical Observations, Palgrave-MacMillan, 2013, pp. 149-172. 
[5] Ł. Bartczuk, P. Dziwiński, J.T. Starczewski, A new method for dealing with unbalanced linguistic term set, in: L. Rutkowski, M. Korytkowski, R. Scherer, R. Tadeusiewicz, L.A. Zadeh, J.M. Zurada (Eds.), Artificial Intelligence and Soft Computing, ICAISC 2012, Lecture Notes in Computer Science 7267, Springer, Berlin, Heidelberg, 2012.

[6] S.M. Baas, H. Kwakernaak, Rating and ranking of multiple-aspect alternatives using fuzzy sets, Automatica 13 (1977) 47-58.

[7] S.J. Chen, C.L. Hwang, Fuzzy Multiple Attribute Decision Making: Methods and Applications, Lecture Notes in Economics and Mathematical Systems, Springer, New York, 1992.

[8] E. Falcó, J.L. García-Lapresta, L. Roselló, Aggregating imprecise linguistic expressions, in: P. Guo, W. Pedrycz (Eds.), Human-Centric DecisionMaking Models for Social Sciences, Springer-Verlag, Berlin, 2014, pp. 97113.

[9] F. Franceschini, M. Galetto, M. Varetto, Qualitative ordinal scales: the concept of ordinal range, Quality Engineering 16 (2004) 515-524.

[10] F. Franceschini, D. Romano, Control chart for linguistic variables: a method based on the use of linguistic quantifiers, International Journal of Production Research 37 (1999) 3791-3801.

[11] J.L. García-Lapresta, Reciprocal and linguistic preferences, in: R. Seising, E. Trillas, C. Moraga, S. Termini (Eds.), On Fuzziness. A Homage to Lotfi A. Zadeh, vol. 1, Springer-Verlag, Berlin, 2013, pp. 193-197.

[12] J.L. García-Lapresta, R. González del Pozo, An ordinal multi-criteria decision-making procedure in the context of uniform qualitative scales, in: M. Collan, J. Kacprzyk (Eds.), Soft Computing Applications for Group Decision-making and Consensus Modeling, Springer, forthcoming.

[13] J.L. García-Lapresta, R. González del Pozo, D. Pérez-Román, Metrizable ordinal proximity measures and their aggregation, submitted.

[14] J.L. García-Lapresta, D. Pérez-Román, Ordinal proximity measures in the context of unbalanced qualitative scales and some applications to consensus and clustering, Applied Soft Computing 35 (2015) 864-872. 
[15] F. Herrera, E. Herrera-Viedma, L. Martínez, A hierarchical ordinal model for managing unbalanced linguistic term sets based on the linguistic 2-tuple model, Proceedings of the EUROFUSE Workshop on Preference Modelling and Applications, pp. 201-206, 2001.

[16] F. Herrera, E. Herrera-Viedma, L. Martínez, A fuzzy linguistic methodology to deal with unbalanced linguistic term set, IEEE Transactions on Fuzzy Systems 16 (2008) 354-370.

[17] F. Herrera, L. Martínez, A 2-tuple fuzzy linguistic representation model for computing with words, IEEE Transactions on Fuzzy Systems 8 (2000), $746-752$.

[18] F. Herrera, L. Martínez, An approach for combining linguistic and numerical information based on 2-tuple fuzzy representation model in decision making, International Journal of Uncertainty, Fuzziness and KnowledgeBased Systems 8 (2000) 539-562.

[19] F.O. Karray, C.W. De Silva, Soft Computing and Intelligent Systems Design: Theory, Tools, and Applications, Pearson Education, 2004.

[20] R. Likert, A technique for the measurement of attitudes, Archives of Psychology 140 (1932) 1-55.

[21] L. Magdalena, What is soft computing? Revisiting possible answers, International Journal of Computational Intelligence Systems 3 (2010) 148-159.

[22] L. Martínez, F. Herrera, An overview on the 2-tuple linguistic model for computing with words in decision making: Extensions, applications and challenges, Information Sciences 207 (2012) 1-18.

[23] F.S. Roberts, Measurement Theory, Addison-Wesley Publishing Company, Reading, 1979.

[24] L.A. Zadeh, The concept of a linguistic variable and its application to approximate reasoning - I, Information Sciences 8 (1975) 199-249.

[25] L.A. Zadeh, Some reflections on soft computing, granular computing and their roles in the conception, design and utilization of information/intelligent systems, Soft Computing 2 (1998) 23-25. 